\title{
How Health Care Complexity Leads to Cooperation and Affects the Autonomy of Health Care Professionals
}

\author{
Eric Molleman · Manda Broekhuis · Renee Stoffels • \\ Frans Jaspers
}

Published online: 11 January 2008

(C) The Author(s) 2008

\begin{abstract}
Health professionals increasingly face patients with complex health problems and this pressurizes them to cooperate. The authors have analyzed how the complexity of health care problems relates to two types of cooperation: consultation and multidisciplinary teamwork (MTW). Moreover, they have analyzed the impact of these two types of cooperation on perceived professional autonomy. Two teams were studied, one team dealing with geriatric patients and another treating oncology patients. The authors conducted semi-structured interviews, studied written documents, held informal discussions and observed the teams at work. Consultation was most likely to take place when a patient had multiple problems. However, if these problems were interrelated, i.e. the solution for one problem interfered with solving another, then MTW was favored. The same was true when the available information was equivocal such that there were conflicting interpretations of a problem. How the professionals perceived the relationship between complexity and the need to cooperate depended on their expertise, their occupational background, and their work orientation. Consultation did not affect the professional autonomy of the health care professionals. MTW however did decrease the perceived level of professional autonomy. The extent to which this occurred seemed to depend on the quality of the interpersonal relations within the team. The findings can help in selecting the most appropriate and efficient type of cooperation based on the complexity of a patient's problems. They can also help team leaders to stimulate reflection and feedback processes, and medical trainers to develop competencies among students related to such teamwork behaviors.
\end{abstract}

\footnotetext{
E. Molleman $(\bowtie) \cdot$ M. Broekhuis

Faculty of Management and Organization, University of Groningen, P.O. Box 800, 9700 AV Groningen, The Netherlands

e-mail: h.b.m.molleman@rug.nl

R. Stoffels · F. Jaspers

University Medical Centre Groningen, Groningen, The Netherlands
} 
Keywords Health care complexity · Cooperation · Professional autonomy

\section{Introduction}

Over recent decades, the complexity of health care demand has increased. Due to an aging population for example, patients increasingly have multiple and interrelated problems [11]. On the provider side, a growth in new technologies and evidencebased knowledge is noticeable, and this has led to a considerable increase in advanced and comprehensive diagnoses and treatments [15]. The expansion of knowledge has also contributed to an increased differentiation and specialization of functions within the medical profession. Given these trends it is apparent that, to achieve satisfactory health care outcomes for individual patients, contributions are increasingly required from multiple disciplines with different occupational backgrounds [10]. These contributions have to be coordinated and this requires health care professionals to cooperate. The first goal of the current study is to explore the relationship between the complexity of the health care demands of an individual patient and the cooperative behaviors of medical providers that result from this situation. One might reasonably expect that, overall, higher levels of complexity in health care demand will lead to more intensive modes of cooperation [4]. To build on this broad statement, in this study, we explore in greater detail the relationships between various aspects of complexity and different modes of cooperation such as multidisciplinary teamwork (MTW).

If providers with different occupational backgrounds have to work together then this may seriously affect their professional autonomy [13]. They will have to coordinate their decisions and actions more frequently through dialogue, and this may significantly increase feelings of having less control over their work. It is likely that such cooperation will affect professional autonomy and accountability [1]. In this study, we will therefore look closely at the interrelationships among different modes of cooperation and various facets of professional autonomy.

\section{Relevance of the Research Questions}

The relationship between complexity and cooperation as well as the impact of cooperation on professional autonomy are relevant for at least two reasons. The first is that a fit between the complexity of the health care demand and the intensity of cooperation would indicate a positive trade-off between health care quality and efficiency. MTW can create an arena for dialogue and creative problem-solving through bringing together expertise from different disciplines. This is likely to contribute to a better solution to a complex problem and, therefore, enhance the quality of health care. However, intensive cooperation requires a lot of time and effort and is a very expensive approach to coordination. If a patient with relatively simple problems is discussed during a multidisciplinary team meeting when a less intensive and cheaper form of coordination would have been sufficient this is clearly inefficient and a waste of resources. 
The second reason is that if cooperation lowers the perceived level of professional autonomy, professionals might avoid it. Medical specialists often have a strong sense of autonomy, and concerns that cooperation might reduce their control over their work might limit their willingness to participate in cooperative practices. As individual physicians increasingly specialize in specific subdisciplines, they also develop related identities and domains of knowledge [5]. Although intensive modes of cooperation such as MTW bring about opportunities to solve complex problems, they can also result in different understandings and distinct ideas of exactly what the problem is and where solutions can be found [14]. Cooperation could lead to confrontation between different professional values and identities, and this could result in an implicit or explicit struggle over the best way to treat a patient's problems [7]. Given these dangers, the relationship between the complexity of the health care demand and the actual intensity of cooperation is not as straightforward as it might first appear.

\section{Method}

\section{Definition of Concepts}

We distinguish three aspects of the complexity of health care demand [16]. Firstly, multiple problems refers to a patient's range of problems that is likely to require the skills and knowledge from several medical specialties. The interrelatedness of problems refers to the fact that the problems are likely to be interwoven and that the best solution to one problem may worsen another and, as a consequence, problems have to be considered in a comprehensive way. Ambiguity occurs when the available information is inconsistent or equivocal resulting in multiple and conflicting interpretations of a problem. As a result there is no obvious best solution, and this hinders evidence-based decision-making. Such a situation might arise, for example, when a doctor considers a radical treatment and has to balance this against quality of life issues.

Modes of working together vary along a continuum starting with a low degree of cooperation and ending with a high level of cooperation [6]. Toward the lower end of the scale, consultation is seen as providing information and support to another on request, and as such involves an explicit feedback loop. A much closer form of cooperation, referred to here as multidisciplinary teamwork (MTW), involves care providers with a range of occupational backgrounds collectively discussing a patient leading to collective decision-making and action.

In this paper, we consider three aspects of professional autonomy [3, 9]. Clinical autonomy relates to the authority to diagnose and determine treatment without the involvement of others. Occupational autonomy refers to the domain of knowledge and skills of a professional with a specific occupational background that professionals with other backgrounds respect as the area in which that professional has the exclusive rights to make decisions and to act. Accountability concerns the social environment in which professionals have potentially to justify their decisions [8]. In professional environments, the social context in which professionals have to 
justify their actions is predominantly the occupational group to which they belong. In a situation where MTW is practiced, the social environment is likely to expand to include professionals with other occupational backgrounds.

\section{Settings}

We have studied two teams of health care professionals in detail, each in a different hospital, in the northern part of the Netherlands. We selected these teams on the basis of two criteria: (a) a range of medical specialties should be involved, and it should be a team in a medical field where cooperation is commonplace, and (b) they had to have been functioning as a team for more than 2 years so that our observations would not be distorted by early group-forming processes. Initially, in both hospitals, lists were compiled of all the teams meeting these criteria. We selected an oncology team and a geriatric team for our research since these operate in fields in which multidisciplinary meetings are seen as indispensable aids to communication and coordination between different specialties [12, 14]. Moreover, both areas are confronted with growing populations, adding pressure to use staff efficiently. The geriatric team was part of a university hospital and the oncology team worked in a general hospital. Both teams had been working together for about 3 years.

The geriatric team worked together for 2 days a week in a day-care clinic. The main objectives were to achieve a comprehensive diagnosis for each patient and to agree a plan for further treatment. The geriatric patients were mostly suffering from a combination of mental, physical, and/or social problems. The team, who spent part of their time on this patient group, consisted of 11 persons: the head of the geriatric department, a clinical geriatrician, a geriatrician internist, a resident internal medicine specialist, a psychiatrist, a resident neurologist, a neurologist, a social worker, two specialized nurses, and a psychologist. For each patient, either the clinical geriatrician or the geriatrician internist would function as the chief doctor-in-charge (CDC). During the course of a day in the day-care clinic, the CDC, the resident doctor from the department of internal medicine and one of the nurses meet regularly to exchange information and to decide on any further diagnostic tests that should be completed that same day. At the end of the day, all the available diagnostic information on each patient is integrated. This group of three workers then assesses whether other team members should be consulted for further tests, or whether it is desirable for the entire team to discuss particular issues in their weekly meetings to discuss patient needs.

The oncology team cared for in-patients with a range of cancers who were undergoing various treatment combinations (radiotherapy, chemotherapy, and/or surgery). The ward could accommodate 34 such patients who were in various stages of the disease (diagnostic stage, curative treatment, or palliative treatment). The main objective of the oncology team was to deliver comprehensive and multidisciplinary care. The team had 21 members: two internist oncologists, two hematologists, four specialized nurses, a surgical oncologist, a resident surgeon, a lung specialist, two internal medicine residents, a radiotherapist, a social worker, a dietician, a physiotherapist, a mental care assistant, a clinical chemist, a pharmacist, and a microbiologist. Depending on the type of cancer and the form of treatment, 
each patient was allocated an appropriate medical specialist as their CDC. The resident doctors, supervised by their $\mathrm{CDC}$, and the oncology nurses carry out the treatment and care tasks. The CDC, the resident doctors, and the nurses primarily coordinate activities in an informal and ad hoc way. Once a week, all the team members meet and, in contrast with the geriatric team, the oncology team discusses all the in-patients during these multidisciplinary meetings.

\section{Procedure}

Two members of our research group conducted semi-structured interviews with the members of both teams. Semi-structured interviews provide an opportunity to reformulate or refine questions based on the responses of interviewees, and so obtain more relevant contextual information. Before starting these interviews, we held three introductory discussions with each team (one with the administrative coordinator, one with the medical coordinator, and one with the whole team) in order to get a clearer general picture of the teams' backgrounds, their goals, and their basic ways of working. The semi-structured interviews that followed lasted about one and a half hours with each team member. During each interview, the main concepts underpinning the study and their interrelationships were discussed extensively. To cross-validate the interview data, we studied documents about the formation and the development of the teams as well as annual reports, protocols, and patient flyers. Further, the two interviewers spent several days in the hospitals providing opportunities for informal talks with the team members and to observe the work processes of the teams. The interviews were tape-recorded and transcribed, and the two interviewers analyzed the data using Atlas [2]. To improve reliability, the two interviewers assigned codes independently and then met to compare their coding. Draft reports were discussed with each team for verification purposes, and both teams considered our results to be accurate.

\section{Results}

In both teams, we were able to identify a core group and a peripheral group. In the geriatric team, for example, the core members were the clinical geriatrician, the internist geriatrician, the internal medicine resident doctor, and the specialized nurses. Below, for illustrative purposes, we include some quotes from the interviewees $(\mathrm{G}=$ quote from a member of the geriatric team; $\mathrm{O}=$ quote by a member of the oncology team).

\section{Complexity of Health Care Demand}

Most of the respondents in both teams understood and recognized the three aspects of complexity we were using. Several interviewees emphasized the fact that complexity increases when a patient has multiple highly interrelated problems, such 
as when the treatment of one problem affects others. They indicated that a comprehensive approach is required if problems are interwoven. As one of the members of the oncology team said:

Most of our patients are very ill and it is important to discuss such patients in a larger group. Not only are the medical issues important but also the psychosocial problems. If, for example, a patient is extremely anxious it may be necessary to postpone a certain medical treatment. Then it becomes important to come up with a comprehensive approach. (O)

Ambiguous situations were also recognized as the following quotes illustrate.

It is hard when the diagnosis is not unequivocal, e.g. when separate tests show results that do not fit. Those are the complex cases. (G)

We can do a lot for our patients, but most of our therapies have severe side effects. That is often the problem: how far can we go and how far should we go? $(\mathrm{O})$

\section{Multiple Problems and Cooperation}

Most of the interviewees indicated that multiple problems generally failed to lead to cooperation (in the active sense explained earlier). Rather, in both teams, the dominant practice was that the core members, and more specifically the CDCs, would only consult other team members in a small number of cases. The CDCs, in their interviews, stated that they were quite able to deal with situations involving multiple problems themselves, and considered this to be a basic aspect of their job.

In most cases, I can completely handle the problems of a patient myself. (O)

Obtaining a complete overview of the different problems of a patient - that is what I'm trained for. $(\mathrm{G})$

Occasionally, the existence of multiple problems would lead the CDC or the core group to call in other members of the team for consultation. In such cases, peripheral members would conduct additional tests and feed back the outcomes to the $\mathrm{CDC}$ who would integrate all the available information. The following quote illustrates this.

I [the geriatrician as $\mathrm{CDC}$ ] receive the results and interpretations of the neurological tests from the neurologist. The neurologist has greater in-depth knowledge and informs me about his conclusions, after which I have to synthesize all the pieces of information and draw my conclusions. (G)

Within the oncology department, multiple problems would lead the CDC to consult other medical specialists within the team directly on a one-to-one basis. Such consultations were mostly determined by protocols and procedures.

Through protocols we know when we need to consult a colleague; we only need to discuss the exceptions during the meeting. (O) 
The responses show that multiple problems do not generally lead to cooperation and, if cooperation is required, it is usually achieved through consultation. Moreover, several respondents indicated that the relationship between multiple problems and cooperation was influenced by the characteristics of the professionals themselves. More specifically, they referred to experience, breadth of expertise domain, and occupational background. With respect to experience, one interviewee stated:

The more experienced you become, the easier it is to deal with more complex

problems yourself. $(\mathrm{O})$

The relevance of the breadth of the physician's domain can be exemplified by the position of the geriatrician. Geriatricians are trained to deal with patients with problems in several areas that previously belonged to different occupational groups such as internal medicine, psychiatry, and neurology. If there is such a range of problems then the geriatrician is able to deal with them autonomously and so will less frequently need to consult others.

The occupational background played a role in the oncology team. It was rare that a non-medical peripheral member (such as the social worker) would be consulted unless a patient was discussed during the multidisciplinary meeting. These nonmedical members believed that they had a more holistic and less mono-disciplinary orientation than the medical specialists in their team and, as a consequence, they tended to signal more social and psychological problems than the medical specialists.

\section{Interrelatedness and Cooperation}

When patients had interrelated problems, we encountered various practices within the two teams. In the geriatric team, some patients were discussed intensively but only within the core medical group. The core group discussed the 'what', 'how', and 'when' issues, leading to a joint decision. Some patients with interrelated problems were, however, then discussed further by the whole team. This was done in a structured way, i.e. the core group formulated specific questions for the larger group in advance, and all the participants received the relevant information (such as test results) before the meeting. Consequently, all the members were able to prepare for the meeting and this facilitated a comprehensive discussion of the patient. Such practices clearly reflect MTW.

In the oncology team, all the patients were put on a list and discussed during the weekly meetings of the entire team. No pre-selection was made although some patients were discussed at greater length than others. This discussion of all the patients was perceived as being inefficient by several team members.

In both teams there were respondents who argued that the perceived interrelatedness of problems was related to characteristics of the professionals involved such as work-related attitudes and occupational background. For example, as noted earlier, the non-medical members of the oncology team were more likely to spot social and psychological problems that could interfere with the medical treatment. 
Consequently, these team members felt a general need for MTW while others preferred to rely on consultations or to avoid cooperation completely. From this, we conclude that while interrelated problems will, in general, result in MTW, the relationship between these two factors depends on the characteristics of the individual health workers involved.

\section{Ambiguity and Cooperation}

The interviews clearly showed that patients in both groups whose problems were perceived as ambiguous were always considered by the entire team, resulting in discussions and collective decision-making. The relationship between ambiguity and MTW is illustrated in the following quotes.

When a patient has been through a long diagnostic route, and it is still not clear which diagnosis is exactly the right one, nobody should have the illusion that they know the one-best-way. In such cases we really do have to meet as a team, and come to a common view and find consensus on how to proceed. $(\mathrm{G})$

In terms of palliative treatment we have several therapies to lengthen life, or to combat symptoms such as pain. However, these interventions often have undesirable side effects. To find the right balance and to come to the best decision requires an open discussion within the team. $(\mathrm{O})$

From this, we conclude that ambiguity leads to MTW, and that the multidisciplinary meeting appears to be an appropriate forum for this.

\section{Cooperation as it Influences Clinical Autonomy}

The interviewees indicated that when they consulted other team members, or when they were themselves consulted by other team members, their clinical autonomy was not affected.

When the CDC decides to consult me, I can autonomously decide how and when I do what. I simply feed back my findings to him. (G)

With respect to $M T W$, we found that within the core groups there was a high level of mutual adjustment, and that this limited the opportunities for autonomous decision-making by individuals. The CDCs, however, indicated that the impact of such activities on their clinical autonomy was rather limited as, in the end, they generally made the final decisions. Nevertheless, several CDCs did indicate that their clinical autonomy was reduced when patients were discussed during meetings of the entire team, even if they did apparently make the final decision.

I notice, if I am honest, that the ideas of other members are often as good as mine or even better. Consciously, or unconsciously, this affects the way I think, decide, and act. (G) 
Discussing patients during multidisciplinary meetings restricts my freedom to make decisions alone. $(\mathrm{O})$

We therefore conclude that consultation does not significantly affect clinical autonomy. MTW, on the other hand, seems to reduce clinical autonomy. In addition to these basic findings, several interviewees suggested interpersonal variables that might influence the relationship between cooperation and clinical autonomy.

It sometimes happens that, even though I am competent to do a certain test, I ask the neuro-psychologist to do it. Not consulting him would make him feel overlooked, which might worsen our interpersonal relationship and impair future cooperation. $(\mathrm{G})$

Over recent years our team has grown. Now, we know each other much better and trust each other much more. Due to this mutual trust, the reluctance to involve others in decision-making has largely disappeared. $(\mathrm{G})$

\section{Cooperation as it Affects Occupational Autonomy}

Our interview data indicate that consultation does not seriously impact on occupational autonomy. In fact, several of the interviewees indicated that being consulted reinforced their sense that their occupational domain was being respected by others. Similarly, consulting others made clear that one's own domain was limited.

Conducting neurological tests is my domain, which is fully respected by the other team members. $(\mathrm{G})$

It is important that you realize where your competence ends and when you have to consult someone else. $(\mathrm{O})$

The impact of $M T W$ on occupational autonomy is more substantial. Most interviewees acknowledged that MTW means that you share information, learn from others, and acquire expertise in the domains of others. As a result, domains of expertise start to overlap and gray areas become more apparent. Some respond to this defensively.

Over the years, the CDC has acquired a great deal of knowledge in my field, and now he carries out a lot of diagnostic activities himself. However, in my opinion, conducting and interpreting neuro-psychological tests is exclusively my competence. If such tests have to be done, others should not question my expertise. (G)

Others responded more favorably to the overlap in expertise domains that resulted from MTW.

You can only work well together if you can accept the fact that others will gain knowledge in your domain. You have to tolerate this, and even stimulate it; otherwise boundary conflicts will arise. (G) 
Other interviewees agreed that MTW led to overlapping domains, and thought that this actually fostered their occupational autonomy.

Through intensive collaboration, others get a better understanding of my field and, as a result, I get greater respect for my expertise. Others listen to me and take me seriously. All this is rewarding for me. $(\mathrm{O})$

We conclude that consultation does not reduce occupational autonomy and can even foster it. MTW seems to affect occupational autonomy more significantly, and medical staff seem to respond to this in individualistic ways. Further, we were made aware of interpersonal variables that might influence the relationship between MTW and the way occupational autonomy is perceived.

In a multidisciplinary team, if you don't trust each other, domain conflicts will easily emerge. (O)

\section{Cooperation Influences Professional Accountability}

Most interviewees indicated that consulting others, or being consulted by others does not seriously affect their professional accountability. Their own specialization group remained the primary audience to which they had to justify decisions. Even when $M T W$ was practiced their own occupational group continued to be an important audience. Nevertheless, MTW did enhance feelings of being accountable toward team members with other occupational backgrounds. Moreover, MTW stimulated collective reflection on work.

When we discuss patients in our team, we openly reflect on the choices made by the individual members of the team. $(\mathrm{G})$

The added value of working together is the extra feedback. You have your own internal control as a professional, but there are also others who can add to this. (O)

Further, MTW also stimulated self-reflection. When making decisions, the individual team members were aware that they should be able to later justify these decisions within the multidisciplinary team. They took this into account when making individual decisions.

I know that I should be able to justify my conclusions with good and reasonable arguments. I have to be well prepared for the multidisciplinary meeting. (G)

From our research, we conclude that consultation has no substantial effect on professional accountability whereas MTW makes one feel accountable to the multidisciplinary team. Here too, we found indications that interpersonal variables affect the impact of MTW on professional accountability.

In the early stage of this team's existence, it was not done to criticize one another. Now that trusting relationships have been developed it is much more acceptable to do so. (G) 


\section{Conclusions}

In this explorative study, we have investigated how health care complexity shapes the way hospital staff work together and how such cooperation affects professional autonomy and accountability. The results show that when studying the relationships between the complexity of health care demand and the modes of cooperation it makes sense to distinguish three aspects of complexity: multiple problems, the interrelatedness of problems, and ambiguity. Multiple problems result in either consultation or in a complete lack of any form of cooperative activity. Interrelated problems and ambiguity tend to lead to MTW.

We found several indications that the relationships between the complexity dimensions and the modes of cooperation depend on characteristics of the individual hospital employee involved. Experience appears to diminish the relationship between health care complexity and cooperation. Doctors with considerable experience can manage a relatively high level of complexity themselves, and this diminishes the perceived need to involve others. The breadth of one's occupational domain can also influence the extent of consultation: having a background that integrates disciplines, such as geriatrics, enables one to deal with matters previously requiring a range of medical specialists. This could be interpreted as indicating that integrated functions can contribute to the efficiency of health care.

Since different occupations can have different work orientations this can result in diverse perceptions of complexity and this can lead to differences in the perceived need to cooperate. In this area, the selection of cases to be discussed by the entire team seems a critical issue. A strong identification with one's own specialty or occupational group might result in a bias in selecting patients and, therefore, in suboptimal care. Some of the non-medical members in our study, for example, thought that the work orientation of some of the medical specialists resulted in social and psychological problems not being sufficiently taken into account in determining treatment. This suggests that a way of working is required that enables all team members to raise cases for discussion at multidisciplinary meetings.

With respect to the relationships between the modes of cooperation and professional autonomy, our interview data show that consultation does not substantially weaken clinical and occupational autonomy, and may even foster them. Professional accountability is also unaffected by consultation. In contrast, MTW appears to reduce clinical and occupational autonomy and leads to an increase in professional accountability toward the multidisciplinary team. A higher level of accountability implies the receipt of more feedback and higher levels of self-reflection and, therefore, enhanced professionalism. Even if there are only very limited evidencebased multidisciplinary guidelines, MTW can still act as a brake on clinicians relying solely on accumulated personal experiences. Based on this, we would argue that multidisciplinary meetings and inter-subjective consensus seem to be the best way to deal with interrelated or ambiguous problems. The link between cooperation and professional autonomy appears to be dependent on characteristics, such as mutual trust, of the interpersonal relationships involved.

All our findings are summarized in Fig. 1. We would emphasize that our study was an explorative one and that the model presented in Fig. 1 is therefore tentative. 


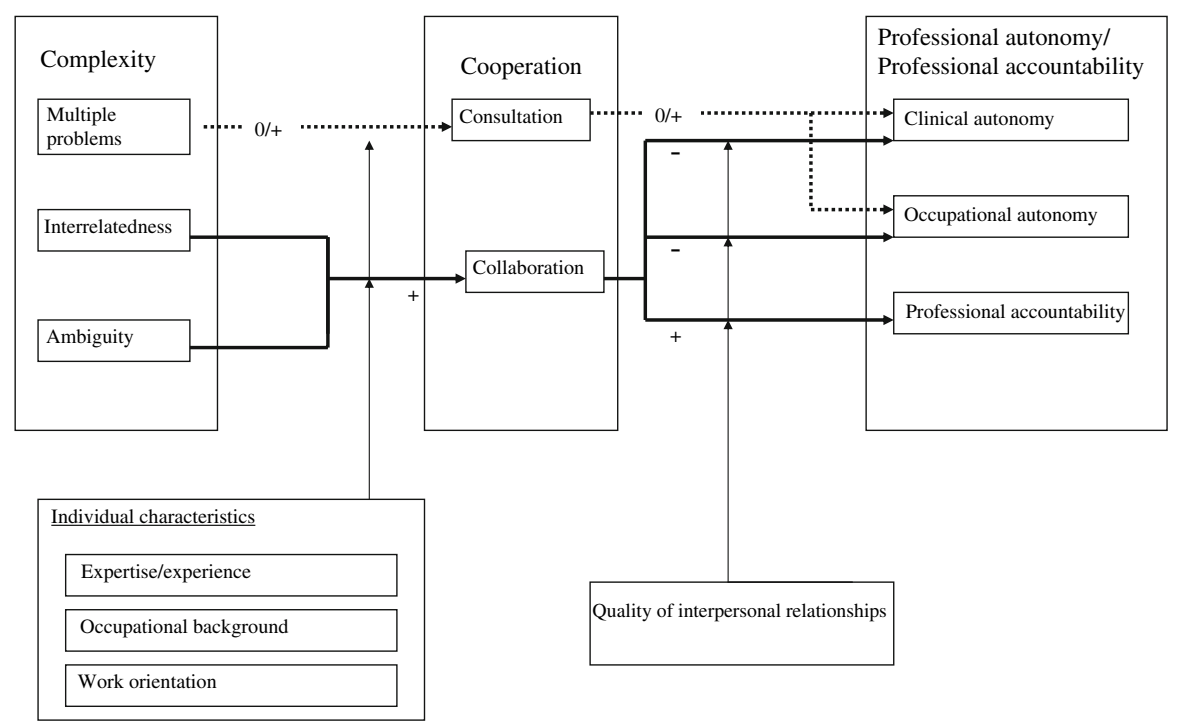

Fig. 1 Relationships found (+ positive relationship, 0 no relationship; - negative relationship)

However, we do believe that it has practical value and so we now proceed to some practical implications.

The facets of complexity that we have distinguished offer a potentially useful framework for developing an instrument to determine health care complexity. Such an instrument could help hospitals to select those patients that should be discussed in a multidisciplinary team and, in so doing, optimize the use of their staff.

MTW seems to reduce clinical autonomy, and this can be a reason for individual professionals to avoid multidisciplinary teamwork. This could increasingly become a serious problem given that the pressure for cooperation is likely to increase as the complexity of patients' problems increases [15]. Our study suggests that the attitudes of professionals towards MTW depend on individual characteristics. This finding could be used in selecting medical students and specialists for training in highly complex fields involving much multidisciplinary work. It could well be beneficial to determine an applicant's attitude towards MTW, and to select those with a positive attitude, rather than only consider more obvious characteristics such as knowledge and technical skills. Our findings may also help team leaders to understand why individual professionals respond differently to MTW demands. These different responses will affect interpersonal relationships and group dynamics, and an insight into these dynamics will help team leaders to improve team effectiveness and to manage or prevent conflicts.

The higher level of accountability linked to MTW results in receiving more feedback and involves higher levels of self-reflection. This requires health professionals who are able to reflect on their own performance, are open to feedback, and are competent in considering their own motives and rationalities and the perspectives of others in a comprehensive way. Since it appears likely that the need for MTW will increase, such competences should be a major concern for those 
who train, select, and manage health professionals [12]. It is also important that team leaders are able to create or support a work climate in which giving and receiving feedback and reflecting on ones own and others' behavior is favored or at least tolerated.

More generally, we believe that our conceptualizations and findings can help health care professionals, team leaders, students, and those who train them, to approach the pains and gains of working in a multidisciplinary team in a more analytical way.

Acknowledgements Funding: This study was supported by ZonMw/NWO, through grant number 5000.0007. We would like to thank R.O.B. Gans, P. Joosten, J.P.J. Slaets, M.A. Verkerk, T. Wiggers and A. van den Wildeberg for their advice and support.

Open Access This article is distributed under the terms of the Creative Commons Attribution Noncommercial License which permits any noncommercial use, distribution, and reproduction in any medium, provided the original author(s) and source are credited.

\section{References}

1. Allison, A., \& Ewens, A. (1998). Tensions in sharing client confidences while respecting autonomy: Implications for inter-professional practice. Nursing Ethics, 5, 441-450.

2. Atlas.ti. (1994). The knowledge workbench; visual qualitative data analysis. http://www.atlasti.de.

3. Breaugh, J. A. (1985). The measurement of work autonomy. Human Relations, 38, 551-570.

4. Brown, T. M., \& Miller, C. E. (2000). Communication networks in task-performing groups. Effects of task complexity, time pressure, and interpersonal dominance. Small Group Research, 31, 131-157.

5. Cott, C. (1997). 'We decide, you carry it out': A social network analysis of multidisciplinary longterm care teams. Social Science \& Medicine, 45, 1411-1421.

6. Doherty, W. J. (1995). The whys and the levels of collaboration. Family Systems Medicine, 13, 275-281.

7. Ferlie, E., Fitzgerald, L., Wood, M., \& Hawkins, C. (2005). The nonspread of innovations: The mediating role of professionals. Academy of Management Journal, 48, 117-134.

8. Frink, D. D., \& Klimoski, R. J. (1998). Toward a theory of accountability in organizations and human resource management. In G. R. Ferris (Ed.), Research in personnel and human resources management (Vol. 16, pp. 39-52). Greenwich: JAI Press.

9. Harrison, S., \& Ahmad, W. I. U. (2000). Medical autonomy and the UK state 1975 to 2025. Sociology, 34, 129-146.

10. Heinemann, G. D., \& Zeiss, A. M. (Eds.). (2002). Team performance in health care. New York: Kluwer Academic Publishers.

11. Hudson, B. (2002). Interprofessionality in health and social care: The Achilles' heel of partnership? Journal of Interprofessional Care, 16, 7-17.

12. Leipzig, R. M., Hyer, K., Ek, K., et al. (2002). Attitudes toward working on interdisciplinary healthcare teams: A comparison by discipline. Journal of the American Geriatrics Society, 50, 1141-1148.

13. Rafferty, A. M., Ball, J., \& Aiken, L. H. (2001). Are teamwork and professional autonomy compatible, and do they result in improved hospital care? Quality in Health care, 10, 32-37.

14. Ruhstaller, T., Roe, H., Thurlimann, B., \& Nicoll, J. J. (2006). The multidisciplinary meeting: an indispensable aid to communication between different specialties. European Journal of Cancer, 42, 2459-2462.

15. Wilson, T., Holt, T., \& Greenhalgh, T. (2001). Complexity and clinical care. BMJ, 323, 685-688.

16. Wood, R. E. (1986). Task complexity: Definition of the construct. Organizational Behavior and Human Decisions Processes, 37, 60-82. 